\title{
Peripheral neuropathy outcomes and efficacy of subcutaneous bortezomib when combined with thalidomide and dexamethasone in the treatment of multiple myeloma
}

\author{
HONG LIU ${ }^{1}$, RUIRONG XU ${ }^{2}$ and HONGMING HUANG ${ }^{2}$ \\ ${ }^{1}$ Department of Hemopathology, Huai'an First People's Hospital, Huai'an, Jiangsu 223300; \\ ${ }^{2}$ Department of Hematopathology, Affiliated Hospital of Nantong University, Nantong, Jiangsu 226001, P.R. China
}

Received March 24, 2015; Accepted August 8, 2016

DOI: $10.3892 / \mathrm{etm} .2016 .3738$

\begin{abstract}
Due to the safety, convenience and efficacy of subcutaneous administration of bortezomib (scBor), it is becoming increasingly common to treat multiple myeloma (MM) using this treatment method. The current retrospective study suggested a lower incidence of peripheral neuropathy (PN) outcomes and superior efficacy following treatment with scBor combined with thalidomide and dexamethasone (VTD) in MM when compared with intravenous Bor (ivBor) treatment. The data of 81 patients from the Affiliated Hospital of Nantong University between September 2011 and February 2014 were analyzed, including 37 scBor and 44 ivBor patients administered a median (range) of 5.5 (3-8) and 6 (3-10) chemotherapy cycles, respectively. Adverse events (AEs) were assessed according to the National Cancer Institute (NCI) Common Terminology Criteria for AEs, and response and progression were assessed by the International Myeloma Working Group criteria. Evidence of histopathology using transmission electron microscopy (TEM) was obtained from an in vivo model of adult Sprague Dawley (SD) rats. Following bortezomib-based VTD chemotherapy, patients had achieved very good partial remission or demonstrated no significant difference between the scBor and ivBor treatment groups (75.6 vs. $84.1 \%$, respectively; $\mathrm{P}=0.350$ ). The 1 -year progression-free survival ( 83.8 vs. $84.1 \%$, scBor vs. ivBor; $\mathrm{P}=0.921)$ and 1-year overall survival (OS) (91.9 vs. 90.9\%, respectively; $\mathrm{P}=0.926)$ were also similar. $\mathrm{PN}$ rates of all the NCI grades were 51.3 and $61.3 \%(P=0.371)$; grade $\geq 2,35.1$ and $56.8 \%(P=0.052)$; and grade $\geq 3,32.7$ and $20.5 \%(\mathrm{P}=0.015)$ in the subcutaneous and intravenous treatment groups, respectively, which suggests that severe PN may be less common following scBor treatment. There were no severe injection site reactions in the scBor-treated
\end{abstract}

Correspondence to: Mrs. Hongming Huang, Department of Hematopathology, Affiliated Hospital of Nantong University, 20 Xisi Road, Nantong, Jiangsu 226001, P.R. China

E-mail:1h011209@163.com

Key words: peripheral neuropathy, bortezomib, subcutaneous injection, thalidomide, multiple myeloma, VTD group. The incidence of adverse events were comparable between the two groups, including thrombocytopenia, anemia, fatigue and gastrointestinal symptoms such as nausea and vomiting. Furthermore, TEM images of the SD rat sciatic nerves revealed that all rats suffered PN to varying degrees, except the control group, and that the PN of ivBor-treated rats (in the presence and the absence of thalidomide) was more severe than that of scBor-treated rats. It was concluded that a subcutaneous dose of bortezomib of $1.3 \mathrm{mg} / \mathrm{m}^{2}$ may result in a lower incidence and severity of PN, with equivalent efficacy, as a component of combination VTD chemotherapy.

\section{Introduction}

Multiple myeloma is the second most common hematological malignancy, with an incidence of 3.29-4.82 cases per 100,000 individuals a year globally, an incidence that is increasing due to population ageing (1). The proteasome inhibitor bortezomib was, at first use, applied to a relapsed or refractory multiple myeloma $(\mathrm{MM})$ at a $1.3 \mathrm{mg} / \mathrm{m}^{2}$ dose, twice per week (2). It then demonstrated its efficacy via $>10$ years of clinical use $(2,3)$. Bortezomib acts upon multiple myeloma by affecting signaling cascades, leading to a toxic buildup of misfolded proteins $(4,5)$. However, the precise mechanism underlying the action of this agent has yet to be fully elucidated. Intravenous injection was previously the standard way of administering bortezomib (2), but subcutaneous administration is increasingly common in the treatment of MM due to the comparative efficacy and improved safety profile of this method of administration, particularly with regard to the lower incidence and severity of peripheral neuropathy (PN). PN primarily affects the sensory nerves, causing symptoms such as a burning sensation, loss of response to external stimuli, paresthesia, discomfort or neuralgia, interfering with patient quality-of-life (6). Use of a single agent, bortezomib, prolonged the median time of progression of MM from 3 to 9 months $(3,7)$. Furthermore, it has been revealed that bortezomib-based combination chemotherapy has an improved efficacy from that of bortezomib alone (2). However, the resultant PN following combination therapy, which includes agents such as thalidomide, is of key importance to address (8). Thalidomide is popular in MM treatment due to its low cost and efficacy profile; this drug suppresses vascular regeneration, improves immune regulation 
Table I. Patient demographics and number of cases demonstrating specific characteristics.

\begin{tabular}{|c|c|c|}
\hline Characteristic & $\begin{array}{l}\text { Subcutaneous bortezomib } \\
\qquad(\mathrm{n}=37 \text { patients })\end{array}$ & $\begin{array}{l}\text { Intravenous bortezomib } \\
\qquad(\mathrm{n}=44 \text { patients })\end{array}$ \\
\hline Age, years (range) & $63(43-85)$ & $64(36-83)$ \\
\hline Age $\geq 65$ years & $15(40.5 \%)$ & $18(40.9 \%)$ \\
\hline Male patients & $28(75.7 \%)$ & $31(70.5 \%)$ \\
\hline Newly diagnosed patients & $35(94.6 \%)$ & $38(86.4 \%)$ \\
\hline \multicolumn{3}{|l|}{ Myeloma type } \\
\hline $\operatorname{IgG}$ & $15(40.5 \%)$ & $17(38.6 \%)$ \\
\hline $\operatorname{IgA}$ & $11(29.7 \%)$ & $13(29.5 \%)$ \\
\hline $\operatorname{IgD}$ & $0(0.0 \%)$ & $2(4.5 \%)$ \\
\hline $\operatorname{IgM}$ & $2(5.4 \%)$ & $2(4.5 \%)$ \\
\hline Light chain & Lam5 (13.5\%), kap4 (10.8\%) & Lam6 (13.6\%), kap4 (9.1\%) \\
\hline \multicolumn{3}{|l|}{ ISS stage } \\
\hline I-II & $15(40.5 \%)$ & $17(38.6 \%)$ \\
\hline III & $22(59.5 \%)$ & $27(61.4 \%)$ \\
\hline No. patients with lytic bone lesions & $24(64.9 \%)$ & $29(65.9 \%)$ \\
\hline Serum albumin, g/l (range) & $34.3(20-40)$ & $32.5(21-38)$ \\
\hline Plasma $\beta 2$ microglobulin, mg/l (range) & $4.58(2.9-23)$ & $5.77(1.8-26.5)$ \\
\hline No. patients using cyclophosphamide & $13(35.1 \%)$ & $15(34.1 \%)$ \\
\hline Renal insufficiency & $6(16.2 \%)$ & $9(20.5 \%)$ \\
\hline Diabetes & $3(8.1 \%)$ & $5(11.4 \%)$ \\
\hline Diabetic neuropathy, grade 1 & $1(2.7 \%)$ & $2(4.5 \%)$ \\
\hline
\end{tabular}

Data are presented as number of cases (percentage of total cases), or mean value (range), where indicated. Ig, immunoglobulin; ISS, International Staging System.

and interferes with the protective bone marrow microenvironment surrounding myeloma cells, promoting apoptosis of tumor cells. PN resulting from thalidomide treatment is common $(8,9)$. Bortezomib-thalidomide-dexamethasone (VTD) is one of the most common chemotherapy regimens; however, the systemic safety profile, particularly regarding the inevitable $\mathrm{PN}$, requires additional attention. In the present study, the incidence of PN following treatment with subcutaneous bortezomib and its efficacy when combined with thalidomide was retrospectively analyzed.

\section{Patients and methods}

Patients and methods. The present study was approved by the Ethics Committee of the Affiliated Hospital of Nantong University, Nantong, China, and written informed consent was obtained from the patient or patients' families. The clinical data of 81 patients ( 37 subcutaneously administered and 44 intravenously administered treatment) of newly diagnosed $(n=77)$ or refractory $(n=4)$ MM following 1-3 prior therapies were comprehensively analyzed. These patients were all admitted to the Affiliated Hospital of Nantong University, Nantong, China between September 2011 and February 2014. All patients received a 21-day cycle of $1.3 \mathrm{mg} / \mathrm{m}^{2}$ bortezomib (Takeda Oncology, Cambridge, MA, USA) on days 1, 4, 8 and 11 by subcutaneous injection (scBor) or intravenous infusion (ivBor), 75-125 mg thalidomide (Changzhou Pharmaceutical Factory, Jiangsu, China) daily and $20 \mathrm{mg}$ dexamethasone (Wuhan
Yuancheng Pharmaceutical Co., Ltd., Hubei, China) on days 1,2, $4,5,8,9,11$ and 12. Neuroprotective therapy, including fursultimine or mecobalamine was administered upon the development of PN. The patients were administered the recommended scBor concentration (3) of $2.5 \mathrm{mg} / \mathrm{ml}(1.75 \mathrm{mg}$ bortezomib reconstituted with $0.7 \mathrm{ml}$ normal $0.9 \%$ saline) or $1.0 \mathrm{mg} / \mathrm{ml}$ ivBor. The subcutaneous injection sites were the thighs and the abdomen, regularly switching side of injection to prevent injection site reaction (ISR). All patients provided written informed consent. Baseline demographics, disease characteristics and chemotherapy regimens were comparable between the two treatment groups (Table I; P>0.05 for all baseline demographics).

The response and progression were assessed by the International Myeloma Working Group criteria (10). Adverse events (AEs) were assessed according to the National Cancer Institute Common Terminology Criteria for AEs version 3.0 (11). The primary aim of this was to investigate the comparative incidence of PN, and the comparative efficacy, following treatment with scBor and ivBor. Grading of neuropathic pain, a symptom of PN, was as follows: Grade 1, pain not interfering with functioning; grade 2 , moderate pain, in which pain or analgesics are interfering with functioning, but not interfering with activities of daily living (ADL); grade 3, severe pain, in which pain or analgesics are severely interfering with ADL; grade 4, disabling pain (11). Efficacy was predominantly determined by the complete remission rate or very good partial remission (CR/VGPR) (10), progression-free survival and overall survival rate (OS) after a median follow-up time of 
Table II. Hematology parameters and rate of other adverse events, expressed as no. cases (\% of total cases).

\begin{tabular}{|c|c|c|c|c|}
\hline \multirow[b]{2}{*}{ Side effects } & \multicolumn{2}{|c|}{$\begin{array}{l}\text { Subcutaneous bortezomib } \\
\qquad(\mathrm{n}=37 \text { patients })\end{array}$} & \multicolumn{2}{|c|}{$\begin{array}{l}\text { Intravenous bortezomib } \\
\quad(\mathrm{n}=44 \text { patients })\end{array}$} \\
\hline & All grades & Grade $\geq 3$ & All grades & Grade $\geq 3$ \\
\hline \multicolumn{5}{|l|}{ Hematology laboratory data } \\
\hline White blood cell count & $19(51.4 \%)$ & $2(5.4 \%)$ & $20(45.5 \%)$ & $9(20.5 \%)$ \\
\hline Hemoglobin level decrease & $28(75.7 \%)$ & $17(45.9 \%)$ & $35(79.5 \%)$ & $22(50.0 \%)$ \\
\hline Platelet count decrease & $17(45.9 \%)$ & $9(24.3 \%)$ & $26(59.1 \%)$ & $17(38.6 \%)$ \\
\hline Diarrhea & $4(10.8 \%)$ & $0(0.0 \%)$ & $5(11.4 \%)$ & $0(0.0 \%)$ \\
\hline Abdominal bloating & $4(10.8 \%)$ & $0(0.0 \%)$ & $7(15.9 \%)$ & $0(0.0 \%)$ \\
\hline Nausea/vomiting & $7(18.9 \%)$ & $0(0.0 \%)$ & $9(20.5 \%)$ & $0(0.0 \%)$ \\
\hline Constipation & $13(35.1 \%)$ & $0(0.0 \%)$ & $15(34.1 \%)$ & $2(4.5 \%)$ \\
\hline Fatigue & $17(45.9 \%)$ & $2(5.4 \%)$ & $29(65.9 \%)$ & $3(6.8 \%)$ \\
\hline Fever & $7(18.9 \%)$ & $0(0.0 \%)$ & $10(22.7 \%)$ & $2(4.5 \%)$ \\
\hline
\end{tabular}

13 (6-16) and 16 months (3-19) in the scBor and ivBor groups, respectively.

Sprague Dawley (SD) rat model. Due to an absence of evidence of PN histopathology in patients, an in vivo model of PN was established in adult SD rats. A total of $18 \mathrm{SD}$ female rats (weight, $200 \pm 5$ g; age, 2 months; sourced from the Animal Laboratory, Nantong University, Nantong, China) were randomly assigned to 6 groups of 3 rats as follows: i) scBor; ii) ivBor; iii) scBor combined with thalidomide; iv) ivBor combined with thalidomide; v) thalidomide only; and vi) untreated control group. These rats were maintained at $24^{\circ} \mathrm{C}$ with a $12 / 12 \mathrm{~h}$ light:dark cycle, and were administered food and water at regular intervals. A dose of $1.3 \mathrm{mg} / \mathrm{m}^{2}(0.2 \mathrm{mg} / \mathrm{kg}$ ) bortezomib (Takeda Oncology) was administered to the relevant groups on days $1,4,8$ and 11 , and $100 \mathrm{mg}(10.5 \mathrm{mg} / \mathrm{kg}$ ) thalidomide (Changzhou Pharmaceutical Factory) was administered daily to the relevant groups. Bortezomib was administered via subcutaneous abdominal or tail vein injection, and thalidomide was administered orally. On the 14th day of treatment, rats were sacrificed by cervical dislocation and sciatic nerve samples were extracted by the Animal Laboratory, Nantong University,. These were examined to determine changes with transmission electron microscopy (TEM).

Statistical methods. Data analysis was performed with the Statistical Package for Social Science software (SPSS version 17.0; SPSS, Inc., Chicago, IL, USA). Continuous variables are expressed as median and range and were compared using a t-test. Categorical variables are reported as percentages and compared using a $\chi^{2}$ test. Overall survival rate, determined from diagnosis to mortality, was estimated by the Kaplan-Meier method and curve comparison was conducted using log-rank analysis. $\mathrm{P}<0.05$ was considered to indicate a statistically significant difference.

\section{Results}

The median number of cycles of bortezomib administered to patients were $5.5(3-8)$ and $6(3-10)$ in the scBor and
ivBor groups, respectively. This included 33 of 37 (89.2\%) scBor-treated patients that had completed $\geq 4$ cycles of treatment, with comparable numbers of 40 of 44 (90.9\%) patients administered ivBor. Patients who had PN or neuropathic pain of grade $\geq 2$ (10) at baseline were excluded from the study. Among the 37 and 44 participating patients, $28(75.6 \%)$ and $37(84.1 \%)$ achieved VGPR or better $(\mathrm{P}=0.350)$, including $13(35.1 \%)$ and 17 (38.6\%) with CR, from the scBor and ivBor groups, respectively $(\mathrm{P}=0.749)$. The median time to reach VGPR was 2 months in both groups. At the final time point, 3 years after the study commencement, 34 (91.9\%) and 40 (90.9\%) patients survived for $\geq 1$ year $(\mathrm{P}=0.926$; Fig. 1$)$ and $31(83.8 \%)$ and 37 (84.1\%) reached 1-year progression-free survival $(\mathrm{P}=0.921$; Fig. 2), in the scBor and ivBor groups, respectively. It is of note that 6 patients ( $2 \mathrm{scBor}$ and 4 ivBor) succumbed to MM by the end of the study. These data collectively suggest an equivalent efficacy of bortezomib administered by either route.

Following supplementation with thalidomide and dexamethasone, the incidence of PN, the most detrimental adverse event, arising due to the treatment remained lower in the scBor-treated group than the ivBor-treated group (all cases: 51.3 vs. $61.3 \%, \mathrm{P}=0.371$; grade $\geq 2: 35.1$ vs. $56.8 \%, \mathrm{P}=0.052$; grade $\geq 3: 2.7$ vs. $20.5 \%, P=0.015$, respectively; Fig. 3). Following treatment with thalidomide, the median time until onset of any grade of PN was 3.0 (1-7) and 4.0 (1-10) months, median time until onset of grade $\geq 2$ was 3.5 (2-11) and $4.0(2-12)$ months in the scBor and ivBor groups, respectively, and time until the onset of grade $\geq 3$ PN was 5.5 (4-10) months in the ivBor group (as no patients demonstrated grade $\geq 3 \mathrm{PN}$ in the scBor group). In the scBor and ivBor groups, 28 (75.7\%) and $29(65.9 \%)$ patients, respectively, were additionally administered fursultimine or mecobalamine upon the development of PN. A total of 0 and 3 (6.8\%) patients in the scBor and ivBor groups, respectively, discontinued thalidomide use or were administered a reduced dose of $50 \mathrm{mg}$ thalidomide for $\sim 1$ month to prevent or attenuate severe PN. Other common side effects were thrombocytopenia, anemia and feeble and gastrointestinal symptoms, including nausea and vomiting (Table II). 


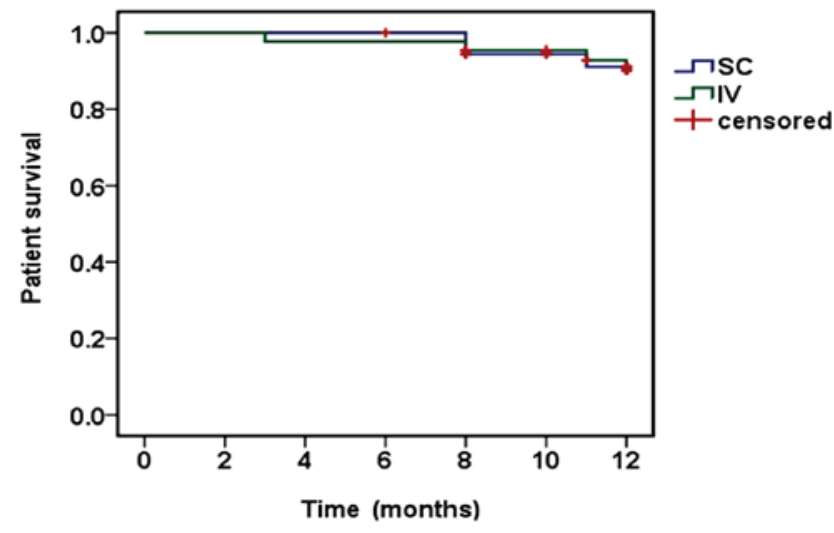

Figure 1. Kaplan-Meier estimates of overall survival following SC and IV bortezomib treatment. SC, subcutaneous; IV, intravenous.

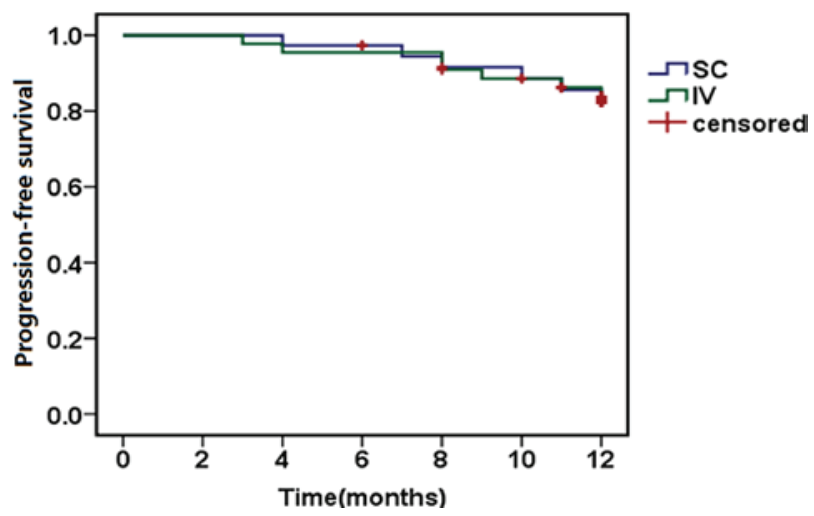

Figure 2. Kaplan-Meier estimates of progression-free survival following SC and IV bortezomib treatment. SC, subcutaneous; IV, intravenous.

Adult SD rats subjected to ivBor treatment gained weight more slowly (Fig. 4) and were less active compared with the untreated control group, in both the presence and absence of thalidomide treatment, indicating systemic poisoning. There were no evident symptoms of systemic poisoning in the scBor-treated and control groups of rats. TEM images indicated PN due to the layered structures (Fig. 5A and B), discontinuous (Fig. 5C) or ruptured tissue (Fig. 5D) of the myelin sheath and vacuolation (Fig. 5E). Furthermore, these observations were more common in the ivBor-treatment group than the scBor group, in both the presence and absence of thalidomide. In addition, only minor changes to the dorsal root nerve of scBor-treated rats were observed.

\section{Discussion}

The present study revealed the superior efficacy, and lower incidence and severity of PN following treatment with scBor compared with ivBor when combined with thalidomide and dexamethasone. Despite several minor differences between this and previous therapies, the similarity between the scBor and ivBor groups across several efficacy indicators (VGPR, $\mathrm{CR}$ and 1-year overall survival/progression-free survival) was apparent, and revealed a significant improvement compared with a single-agent therapy $(3,7)$. This efficacy may be associated with a higher percentage of patients receiving additional

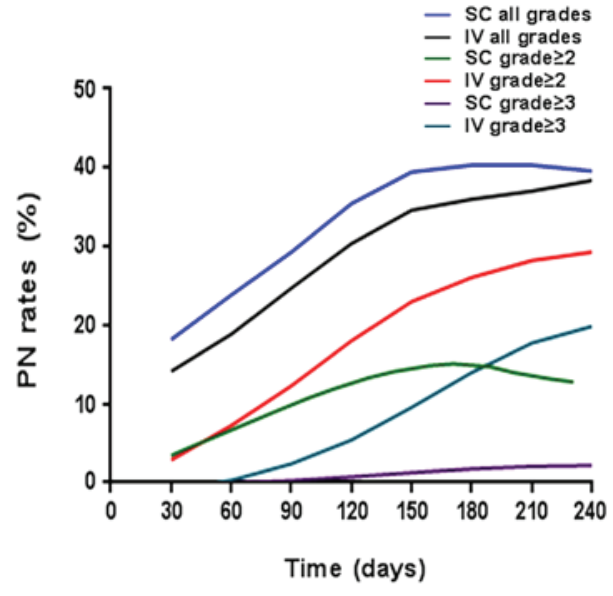

Figure 3. Increasing PN rates following SC and IV bortezomib treatment. $\mathrm{PN}$, peripheral neuropathy; SC, subcutaneous; IV, intravenous.

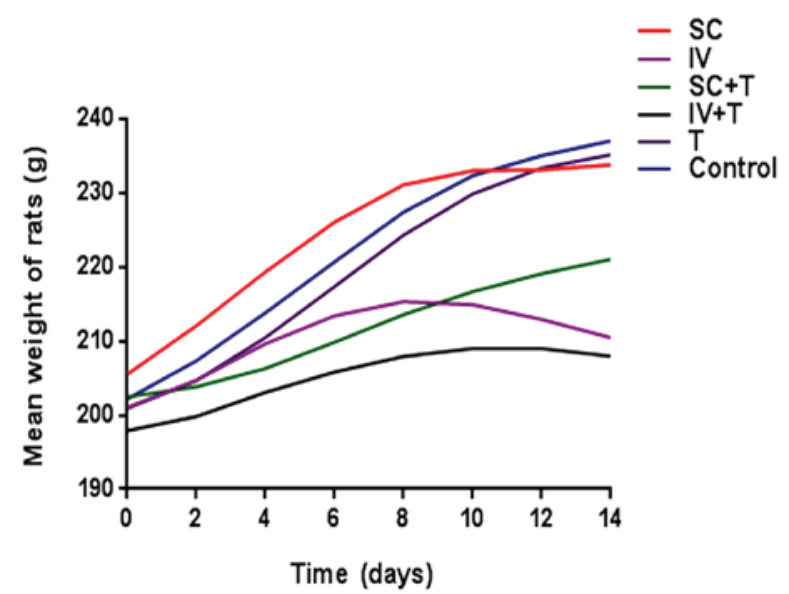

Figure 4. Mean weights of Sprague-Dawley rats in the six groups. SC, subcutaneous; IV, intravenous.

cyclophosphamide, a lower percentage of patients with an ISS stage III (12) or an age $\geq 65$ years old and a lower level of $\beta 2$ microglobulin in the scBor-treated group.

The intravenous method of administration is the most common method of bortezomib treatment. However, subcutaneous injection of bortezomib is becoming increasingly used, and the side effects of scBor single treatment are well understood $(3,7,13,14)$. A previous study by Arnulf et al (7) of treatment with bortezomib alone demonstrated that the incidence of PN at all grades, grade $\geq 2$ and grade $\geq 3$ were 38 and $53 \%, 24$ and $41 \%$ and 6 and $16 \%$ in the scBor and ivBor groups, respectively. This was in agreement with the 51.3 and $61.3 \%, 35.1$ and $56.8 \%$ and 2.7 and $20.5 \%$ observed in the scBor and ivBor groups, respectively, in the present study. Garderet et al (15) demonstrated a cumulative, dose-associated incidence of grade $\geq 3$, exacerbated PN following treatment with bortezomib and thalidomide combinatorial treatment; this presented at an incidence of $29 \%$ for VTD treatment and $12 \%$ for thalidomide and dexamethasone treatment. The median time of onset of all grades of $\mathrm{PN}$ of the patients in the present study was similar, and there were no cases of grade $\geq 3 \mathrm{PN}$ associated with scBor treatment. These data suggested a decrease in severity of PN following scBor treatment, which may be associated with a lower maximum 


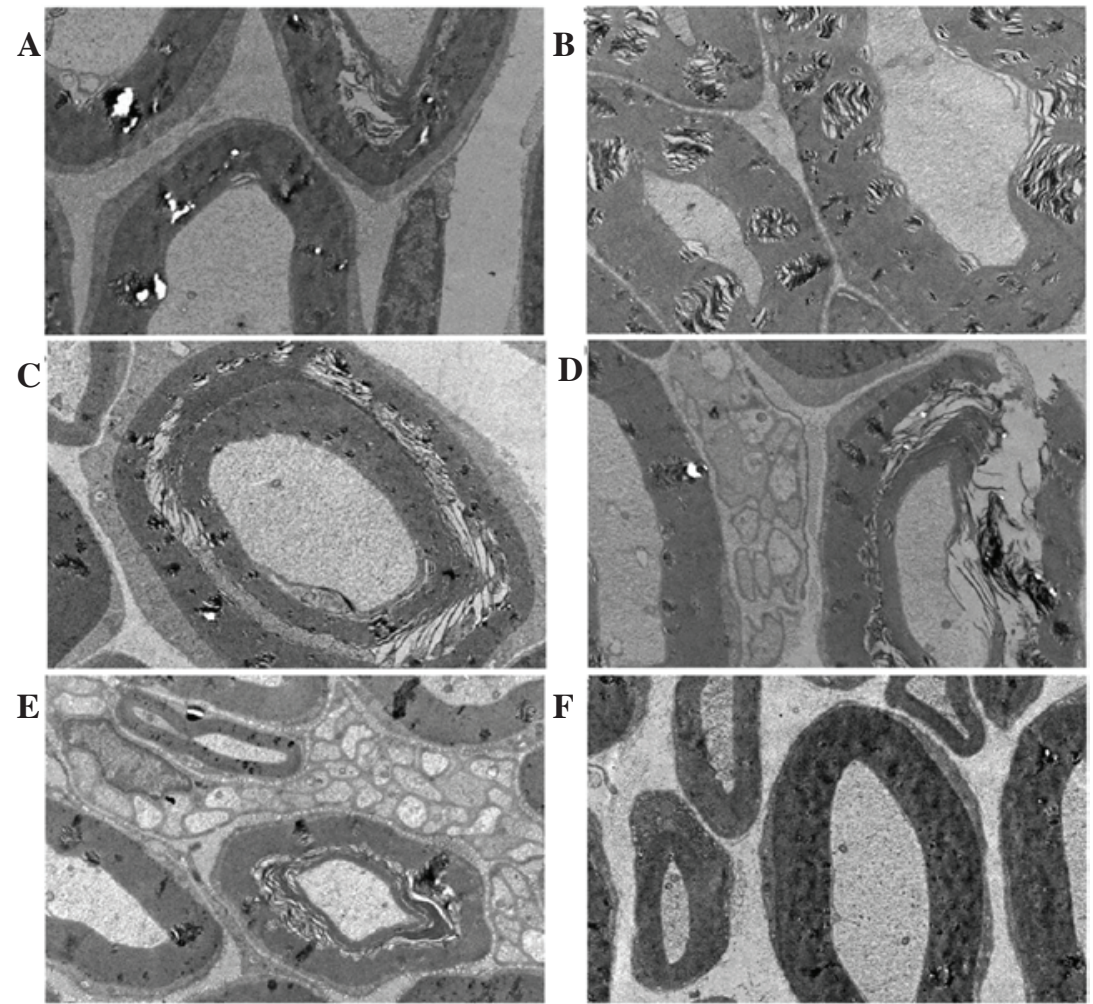

Figure 5. Transmission electron microscope images (magnification, x10,000) of left sciatic nerves in rats from six groups, as follows: (A) scBor; (B) scBor combination treatment with thalidomide; (C) ivBor; (D) ivBor combination treatment with thalidomide; (E) thalidomide only and (F) control. sc, subcutaneous; Bor, bortezomib; iv, intravenous.

plasma concentration of bortezomib, neuroprotective effects and thalidomide withdrawal or with dose reduction when severe PN appeared in 3 cases (3). PN occurred more frequently following subcutaneous delivery of bortezomib, but was less severe following this method of bortezomib treatment. In addition, bortezomib-associated PN typically became apparent by the end of therapy cycles 4 or 5 , was dose-associated and was reversible in the majority of patients. The baseline characteristics of the age or number or type of prior therapies were not risk factors associated with PN, and the efficacy of bortezomib was not adversely affected by the grade $\geq 2 \mathrm{PN}$ (16) or the dose regimen (17). Furthermore, there were no obvious ISRs in the current patients, and the low severity of ISRs was similar to that of a previous report (18). Subcutaneous administration of bortezomib therefore provides an important alternative method of treatment, particularly for the elderly or patients with poor vascular conditions, due to its improved safety profile.

Detailed information with regard to other side effects of bortezomib have been sufficiently documented whether this is administered independently or as part of a combination therapy (18-23). Upon treatment with the VTD combination therapy, a decreased white blood cell count, hemoglobin level and platelet count, and gastrointestinal symptoms were the most common side effects in both groups, but these were not severe in the present study. These data were consistent with previous studies $(22,23)$ and presented additional information about the scBor severity as part of a VTD combination therapy.

From the results of previous in vivo studies, it has been hypothesized that bortezomib predominantly causes direct sciatic nerve and dorsal root ganglia toxicity due to ubiquitin aggregates accumulating in the cytoplasm $(24,25)$. In the current study, TEM images of the sciatic nerve of the SD rats indicated that changes to the myelin sheath were less common following scBor compared with ivBor treatment. Furthermore, $\mathrm{PN}$ is more severe in combination treatments containing thalidomide; however, this effect was lessened with scBor combination compared with the ivBor combination treatment. Symptoms of systemic poisoning, such as a slower weight increase and lower activity, were more evident following ivBor treatment, in both the presence and absence of thalidomide.

In conclusion, treatment with scBor demonstrated a lower incidence and severity of PN compared with ivBor administration, but did not adversely affect the efficacy of treatment when combined with thalidomide and dexamethasone. Previous studies have indicated that a weekly dose of bortezomib should be used within combinatorial treatments in order to achieve the lowest PN rates (26-28). However, the most efficient dose schedule of bortezomib under varying ISS stages and patient demographics requires additional research.

\section{References}

1. Phekoo KJ, Schey SA, Richards MA, Bevan DH, Bell S, Gillett D and Møller H; Consultant Haematologists, South Thames Haematology Specialist Committee: A population study to define the incidence and survival of multiple myeloma in a National Health Service Region in UK. Br J Haematol 127: 299-304, 2004.

2. Pantani L, Zamagni E, Zannetti BA, Pezzi A, Tacchetti P, Brioli A, Mancuso K, Perrone G, Rocchi S, Tosi P and Cavo M: Bortezomib and dexamethasone as salvage therapy in patients with relapsed/refractory multiple myeloma: Analysis of long-term clinical outcomes. Ann Hematol 93: 123-128, 2014. 
3. Moreau P, Pylypenko H, Grosicki S, Karamanesht L, Leleu X, Grishunina M, Rekhtman G, Masliak Z, Robak T, Shubina A et al: Subcutaneous versus intravenous administration of bortezomib in patients with relapsed multiple myeloma: A randomised, phase 3, non-inferiority study. Lancet Oncol 12: 431-440, 2011.

4. Soriano GP, Besse L, Li N, Kraus M, Besse A, Meeuwenoord N, Bader J, Everts B, den Dulk H, Overkleeft HS, Florea BI and Driessen C: Proteasome inhibitor-adapted myeloma cells are largely independent from proteasome activity and show complex proteomic changes, in particular in redox and energy metabolism. Leukemia, 2016.

5. Ri M: Mechanism of action and determinants of sensitivity to the proteasome inhibitor bortezomib in multiple myeloma therapy. Rinsho Ketsueki 57: 537-45, 2016.

6. Dimopoulos MA, Beksac M, Benboubker L, Roddie H, Allietta N, Broer E, Couturier C, Mazier MA, Angermund R and Facon T: Phase II study of bortezomib-dexamethasone alone or with added cyclophosphamide or lenalidomide for sub-optimal response as second-line treatment for patients with multiple myeloma. Haematologica 98: 1264-72, 2013.

7. Arnulf B, Pylypenko H, Grosicki S, Karamanesht L, Leleu X, van de Velde H, Feng H, Cakana A, Deraedt W and Moreau P: Updated survival analysis of a randomized phase III study of subcutaneous versus intravenous bortezomib in patients with relapsed multiple myeloma. Haematologica 97: 1925-1928, 2012.

8. Glasmacher A, Hahn C, Hoffmann F, Naumann R, Goldschmidt H, von Lilienfeld-Toal M, Orlopp K, Schmidt-Wolf I and Gorschlüter M: A systematic review of phase-II trials of thalidomide monotherapy in patients with relapsed or refractory multiple myeloma. Br J Haematol 132: 584-593, 2006.

9. Palumbo A, Davies F, Kropff M, Bladé J, Delforge M, Leal da Costa F, Garcia Sanz R, Schey S, Facon T, Morgan G and Moreau P: Consensus guidelines for the optimal management of adverse events in newly diagnosed, transplant-ineligible patients receiving melphalan and prednisone in combination with thalidomide (MPT) for the treatment of multiple myeloma. Ann Hematol 89: 803-811, 2010.

10. Durie BG, Harousseau JL, Miguel JS, Bladé J, Barlogie B, Anderson K, Gertz M, Dimopoulos M, Westin J, Sonneveld P, Ludwig $\mathrm{H}$ et al: International uniform response criteria for multiple myeloma. Leukemia 20: 1467-73, 2006

11. National Cancer Institute: Common terminology criteria for adverse events, version 3.0. https://ctep.cancer.gov/protocolDevelopment/electronic_applications/docs/ctcaev3.pdf. Accessed April 11, 2011.

12. Greipp PR, San MJ, Durie BG, Crowley JJ, Barlogie B, Bladé J, Boccadoro M, Child JA, Avet-Loiseau H, Kyle RA, Lahuerta JJ et al: International staging system for multiple myeloma. J Clin Oncol 23: 3412-20, 2005.

13. Hoy SM: Subcutaneous bortezomib: In multiple myeloma. Drugs 73: 45-54, 2013.

14. Grosicki S: Subcutaneous bortezomib as a new promising way to successful maintenance therapy in multiple myeloma. Wiad Lek 65: 167-173, 2012 (In Polish).

15. Garderet L, Iacobelli S, Moreau P, Dib M, Niederwieser D, Masszi T, Fontan T, Michallet M, Gratwohl A, Lafon I et al: Superiority of the triple combination of bortezomib-thalidomide-dexamethasone over the dual combination of thalidomide-dexamethasone in patients with multiple myeloma progressing or relapsing after autologous transplantation: The MMVAR/IFM 2005-04 Randomized phase III trial from the chronic leukemia working party of the European group for blood and marrow transplantation. J Clin Oncol 30: 2475-2482, 2012.
16. Tacchetti P, Terragnac C, Galli M, Zamagni E, Petrucci MT, Pezzi A, Montefusco V, Martello M, Tosi P, Baldini L et al: Bortezomib- and thalidomide-induced peripheral neuropathy in multiple myeloma: Clinical and molecular analyses of a phase 3 study. Am J Hematol 89: 1085-1091, 2014.

17. Richardson PG, Sonneveld P, Schuster MW, Stadtmauer EA, Facon T, Harousseau JL, Ben-Yehuda D, Lonial S, Goldschmidt H, Reece D et al: Reversibility of symptomatic peripheral neuropathy with bortezomib in the phase III APEX trial in relapsed multiple myeloma: Impact of a dose-modification guideline. Br J Haematol 144: 895-903, 2009.

18. Lamm W, Drach-Schauer B, Eder S and Drach J: Bortezomib administered subcutaneously is well tolerated in bortezomib-based combination regimens used in patients with multiple myeloma. Oncology 85: 223-227, 2013.

19. San Miguel JF, Schlag R, Khuageva NK, Dimopoulos MA, Shpilberg O, Kroptt M, Spicka I, Petrucci MT, Palumbo A, Samoilova OS et al: Bortezomib plus melphalan and prednisone for initial treatment of multiple myeloma. N Engl J Med 359: 906-917, 2008

20. Wright JJ: Combination therapy of bortezomib with novel targeted agents: An emerging treatment strategy. Clinical Cancer Research 16: 4094-4104, 2010.

21. San Miguel JF, Schlag R, Khuageva NK, Dimopoulos MA, Shpilberg O, Kropff M, Spicka I, Petrucci MT et al: Continued overall survival benefit after 5 years' follow-up with bortezomib-melphalan-prednisone (VMP) versus melphalan-prednisone (MP) in patients with previously untreated multiple myeloma and no increased risk of second primary malignancies: Final results of the phase 3 VISTA trial. Blood 118: 476, 2011.

22. Hrusovsky I, Emmerich B, von Rohr A, Voegeli J, Taverna C, Olie RA, Pliskat H, Frohn C and Hess G: Bortezomib retreatment in relapsed multiple myeloma - results from a retrospective multicentre survey in Germany and Switzerland. Oncology 79: 247-54, 2010.

23. Sher T, Ailawadhi S, Miller KC, Manfredi D, Wood M, Tan W, Wilding G, Czuczman MS, Hernandez-llizaliturri FJ, Hong F et al: A steroid-independent regimen of bortezomib, liposomal doxorubicin and thalidomide demonstrate high response rates in newly diagnosed multiple myeloma patients. $\mathrm{Br}$ J Haematol 154: 104-110, 2011.

24. Cavaletti G, Gilardini A, Canta A, Rigamonti L, Rodriguez-Menendez V, Ceresa C, Marmirili P, Bossi M, Oggioni N, D'Incalci M and De Coster R: Bortezomib-induced peripheral neurotoxicity: A neurophysiological and pathological study in the rat. Exp Neurol 204: 317-325, 2007.

25. Csizmadia V, Raczynski A, Csizmadia E, Fedyk ER, Rottman J and Alden CL: Effect of an experimental proteasome inhibitor on the cytoskeleton, cytosolic protein turnover and induction in the neuronal cells in vitro. Neurotoxicology 29: 232-243, 2008.

26. Mangiacavalli S, Pochintesta L, Pascutto C, Cocito F, Cazzola M, Corso A and Pompa A: Good clinical activity and favorable toxicity profile of once weekly bortezomib, fotemustine and dexamethasone (B-MuD) for the treatment of relapsed multiple myeloma. Am J Hematol 88: 102-106, 2013.

27. Bringhen S, Larocca A, Rossi D, Cavalli M, Genuardi M, Ria R, Gentili S, Patriarca F, Nozzoli C, Levi A et al: Efficacy and safety of once-weekly bortezomib in multiple myeloma patients. Blood 116: 4745-4753, 2010.

28. Moore S, Atwal S, Sachchithanantham S, Streetly M, Khan I, Percy L, Narat S, Rabin N, Johnston R, D'Sa S et al: Weekly intravenous bortezomib is effective and well tolerated in relapsed/refractory myeloma. Eur J Haematol 90: 420-425, 2013. 